\title{
Neoadjuvant immunotherapy in resectable non-small cell lung cancer at a checkpoint
}

\author{
Claire M. Faltermeier, Jay M. Lee \\ Division of Thoracic Surgery, David Geffen School of Medicine at UCLA, Los Angeles, CA, USA \\ Correspondence to: Jay M. Lee, MD. Division of Thoracic Surgery, Ronald Reagan UCLA Medical Center, Los Angeles, CA 90095-7313, USA. \\ Email: jaymoonlee@mednet.ucla.edu. \\ Comment on: Qiu B, Cai K, Chen C, et al. Expert consensus on perioperative immunotherapy for local advanced non-small cell lung cancer. Transl \\ Lung Cancer Res 2021;10:3713-36.
}

Submitted Oct 12, 2021. Accepted for publication Oct 27, 2021.

doi: $10.21037 /$ tlcr-21-830

View this article at: https://dx.doi.org/10.21037/tlcr-21-830

The authors should be congratulated for developing an expert consensus statement regarding neoadjuvant immune checkpoint inhibition (ICI) in resectable non-small cell lung cancer (NSCLC). However, the current body of evidence is largely based on phase II neoadjuvant ICI alone or with chemotherapy (CT-ICI) trials, and the phase III trials of CT-ICI are ongoing (Table 1) (1). Our objective is to provide an update on the role of induction ICI in early stage NSCLC.

Neoadjuvant ICI has the potential to be practice changing in resectable NSCLC. Phase II neoadjuvant ICI trials demonstrated that ICI (I) is safe with low serious (grade $\geq 3$ ) treatment related adverse events (TRAE), (II) results in few delays to surgery with low pre-operative attrition to resection, (III) has little impact on perioperative morbidity or mortality, and (IV) has therapeutic efficacy (pathologic regression) following induction therapy. However, validation of pathological regression [major pathologic regression (MPR) or pathologic complete response (pCR)] as a predictor of clinical outcomes [disease free survival (DFS), event free survival (EFS), or overall survival (OS)] remains unproven in NSCLC. As such, until there is clinical outcome correlation to pathologic regression, the use of neoadjuvant ICI alone or CT-ICI remains investigational and should not be advocated as standard of care. The focus of clinical trial design has been on therapeutic efficacy and TRAE. However, surgical and clinical endpoints need to be incorporated into prospective data collection and reporting (2). Here, we address each consensus statement with consideration of the current perioperative landscape.

\section{Consensus 1: preoperative use of neoadjuvant immunotherapy with or without platinum-based chemotherapy for patients with resectable stage IB-IIIA NSCLC may be considered}

Neoadjuvant ICI has the potential to improve outcomes in resectable NSCLC. Phase II trials of neoadjuvant ICI alone or with CT have shown no significant safety concerns and pathologic regression was demonstrated $(1,2)$. In LCMC3, the largest neoadjuvant ICI monotherapy trial, the preoperative and postoperative grade $\geq 3$ TRAE following neoadjuvant atezolizumab was $6 \%$ and $11 \%$, respectively (3). CheckMate 816 (CM816) is the first phase III neoadjuvant nivolumab and CT trial to report that grade $\geq 3$ TRAE of $34 \%(4,5)$. In comparison to historical neoadjuvant CT trials, the increased grade $\geq 3$ TRAE with CT-ICI compared to ICI alone is predominantly due to CT $(1,2)$.

Treatment efficacy in neoadjuvant trials has been defined as either MPR $[<10 \%$ viable tumor $(\mathrm{VT})]$ or pCR (absence of VT) (1). MPR and pCR after neoadjuvant ICI alone was $14-45 \%$ and $5-16 \%$, respectively, compared to CT-ICI with $57-85 \%$ and $18-63 \%$, respectively, in NSCLC (1). Although OS is the gold standard efficacy endpoint, time from enrollment to study publication (9-13 years) significantly delays perioperative drug development (1). There is precedent in resectable breast cancer where pCR following neoadjuvant therapy was associated with significant improvement in EFS and OS compared to nonpCR [EFS HR (95\% confidence interval): 0.24 (0.20-0.29) and OS: $0.19(0.15-0.24)]$ (6). However, the relationship between pathologic response and clinical outcome (DFS, 
Table 1 Ongoing phase III trials of neoadjuvant combination chemotherapy and immunotherapy

\begin{tabular}{|c|c|c|c|c|c|c|c|c|c|}
\hline CT.gov ID & Study/site & Status & $\begin{array}{l}\text { Start } \\
\text { date }\end{array}$ & Neoadjuvant therapy & $\mathrm{N}$ & $\begin{array}{l}\text { EGFR or } \\
\text { ALK }\end{array}$ & Adjuvant therapy & Stage & $\begin{array}{l}\text { Primary } \\
\text { endpoint }\end{array}$ \\
\hline NCT03800134 & AEGEAN & Active & 2018 & $\begin{array}{l}\text { Durvalumab + CT vs. } \\
\text { placebo + CT (4 cycles; } \\
12 \text { weeks) }\end{array}$ & 800 & Included & $\begin{array}{l}\text { Durvalumab vs. } \\
\text { supportive care }\end{array}$ & II, III & $\begin{array}{l}\text { MPR, } \\
\text { EFS }\end{array}$ \\
\hline NCT02998528 & $\begin{array}{l}\text { CheckMate } \\
816\end{array}$ & Active & 2017 & $\begin{array}{l}\text { Ipilimumab + nivolumab } \\
\text { vs. nivolumab + CT vs. } \\
\text { CT ( } 3 \text { cycles; } \lessgtr 9 \text { weeks) }\end{array}$ & 350 & Excluded & None & IB-IIIA & $\begin{array}{l}\mathrm{pCR} \\
\text { EFS }\end{array}$ \\
\hline NCT04025879 & $\begin{array}{l}\text { CheckMate } \\
77 \mathrm{~T}\end{array}$ & Active & 2019 & $\begin{array}{l}\text { Nivolumab + CT vs. } \\
\text { placebo + CT ( } 4 \text { cycles; } \\
12 \text { weeks) }\end{array}$ & 452 & Excluded & Nivolumab vs. placebo & $\begin{array}{c}\|\mathrm{A}(<4 \mathrm{~cm})-\| \mathrm{IIB} \\
(\mathrm{T} 3 \mathrm{~N} 2)\end{array}$ & EFS \\
\hline NCT03456063 & $\begin{array}{l}\text { IMpower } \\
030\end{array}$ & Active & 2018 & $\begin{array}{l}\text { Atezolizumab + CT vs. } \\
\text { placebo + CT ( } 4 \text { cycles; } \\
12 \text { weeks) }\end{array}$ & 450 & Excluded & $\begin{array}{l}\text { Atezolizumab ( } 16 \text { cycles) } \\
\text { vs. supportive care }\end{array}$ & II, IIIA, IIIB & $\begin{array}{l}\text { MPR, } \\
\text { EFS }\end{array}$ \\
\hline NCT04379635 & China & Active & 2020 & $\begin{array}{l}\text { Tislelizumab + CT vs. } \\
\text { placebo + CT ( } \lesssim 12 \\
\text { cycles) }\end{array}$ & 380 & Excluded & $\begin{array}{l}\text { Tislelizumab (12 cycles) } \\
\text { vs. placebo }\end{array}$ & $\|-\| I A$ & $\begin{array}{l}\text { MPR, } \\
\text { EFS }\end{array}$ \\
\hline
\end{tabular}

$\mathrm{N}$, number; CT, chemotherapy; MPR, major pathologic response; EFS, event free survival, pCR, pathologic complete response; OS, overall survival.

EFS or OS) has yet to be validated in NSCLC. As a result, presently, the use of ICI or CT-ICI remains investigational until this correlation is made from the ongoing phase III neoadjuvant trials (Table 1) (1).

\section{Consensus 2: there is no evidence that molecular markers uniformly predict the efficacy of neoadjuvant immunotherapy so that biomarker based selection is not essential; however it should be cautious to use neoadjuvant single agent immunotherapy in patients with potentially negative factors such as EGFR and ALK fusion}

Reliable biomarkers predictive of preoperative ICI efficacy are lacking. PD-L1 expression and tumor mutation burden (TMB) have correlated with response to immunotherapy in advanced NSCLC $(7,8)$. In phase II neoadjuvant ICI trials, the association between PD-L1 or TMB to pathologic response have varied (1). Additional studies are needed to define biomarkers in the neoadjuvant setting.

Presence of driver mutations has consistently shown poor responses to immunotherapy. In LCMC3, there were no patients with EGFR or ALK mutations that achieved MPR following neoadjuvant atezolizumab (9). In the phase III IMpower010 study of adjuvant atezolizumab, only tumors with PD-L $1 \geq 1$ for stage II and III NSCLC experienced DFS advantage (10). Tumors expressing driver mutations such as ROS1, BRAF, HER2, MET and RET have historically shown poor responses to ICI with objective response rates (ORR) of 4-23\% (11). Furthermore, the combination of targeted therapy (TT) and immunotherapy is associated with high rates $(>20 \%)$ of grade 3-4 toxicities (12). Additionally, in ADAURA, a phase 3 trial of adjuvant osimertinib in stage IB-IIIA NSCLC with activating EGFR mutations, there was an impressive DFS benefit compared to placebo (13). As such, ICI should be avoided in tumors with activating EGFR or ALK alterations. Given these data, we recommend routine molecular testing of tumors in early stage NSCLC to identify driver mutations. Patients with early-stage driver mutation-positive NSCLC should be considered for neoadjuvant or adjuvant TT trials or treatments. 


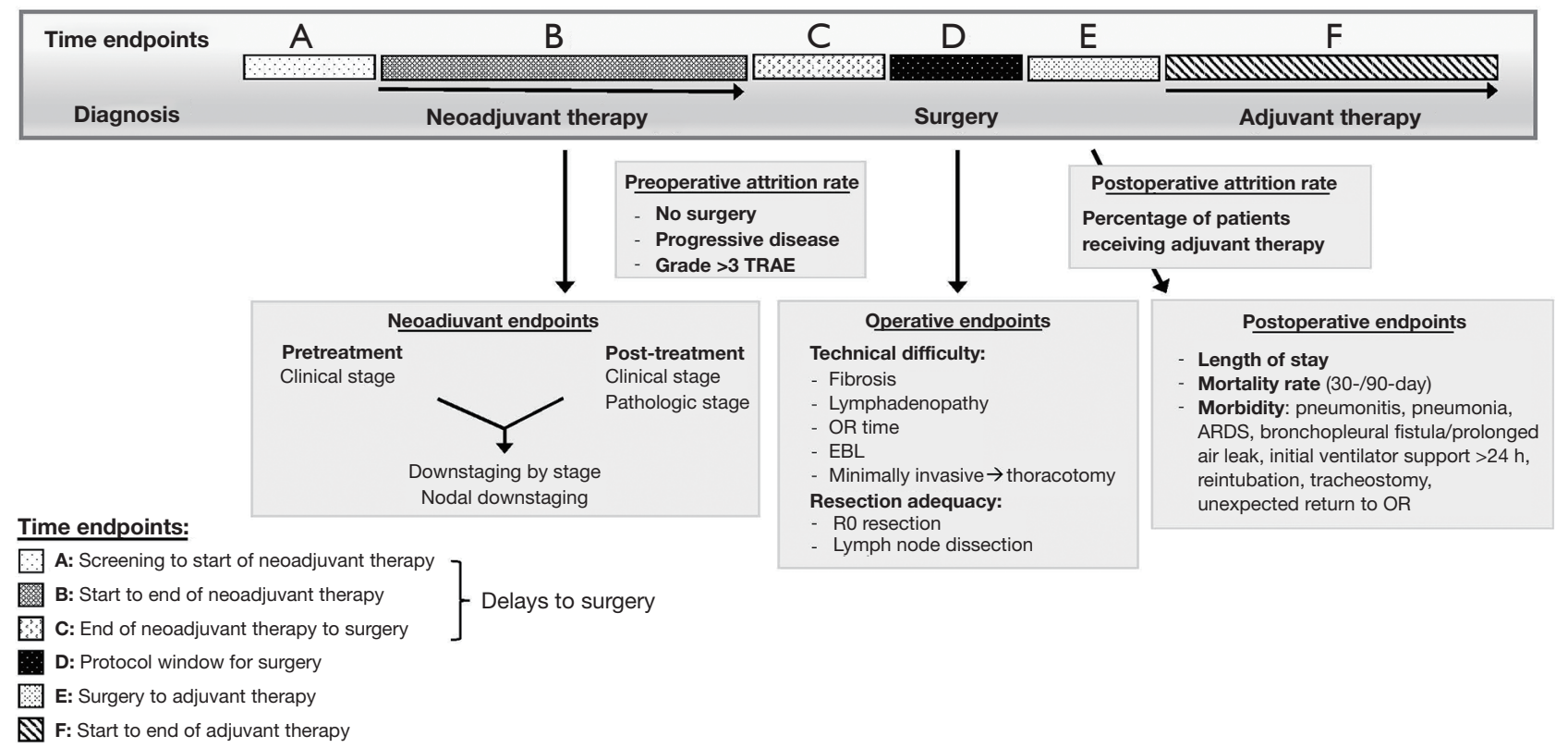

Figure 1 Important surgical and clinical endpoints in neoadjuvant trials*. *, adapted from Lee et al. (2). TRAE, treatment related adverse events; OR, operating room; EBL, estimated blood loss; R0, complete resection; ARDS, acute respiratory distress syndrome.

\section{Consensus 3: for neoadjuvant immunotherapy, two to four cycles are recommended and after every two cycles, review and evaluation should be performed to update the treatment plan}

The optimal number neoadjuvant immunotherapy cycles remains under investigation. Phase III trials of neoadjuvant CT-ICI are ongoing with primary endpoints of MPR, pCR, EFS, or OS (Table 1). In these trials, neoadjuvant regimens range 3-12 cycles with 4 cycles (12 weeks) in four of six phase III trials (Table 1). In LCMC3, 2 cycles of neoadjuvant atezolizumab was given prior to resection. At the current time, there is no established number of ICI cycles.

\section{Consensus 4: the benefit from neoadjuvant immunotherapy should be preferably assessed by PET-CT in conjunction with serum tumor markers and/or circulating tumor DNA load (ctDNA)}

Following neoadjuvant ICI, PET-CT imaging should be performed to assess response and extent of disease. However, the interval between neoadjuvant immunotherapy and surgery is relatively short (Figure 1), limiting the accuracy of radiographic tumor size reduction (RECIST) as a measure of ICI efficacy. In LCMC3, the median time between completion of atezolizumab to surgery was 22 days (time endpoint C; Figure 1) (3). In NEOSTAR (neoadjuvant nivolumab or nivolumab and ipilimumab), there was a positive association between radiographic and pathologic response, but MPR was also observed in stable disease (14). Additionally, radiographically perceived progressive disease with metabolically-avid lymphadenopathy should be cautiously interpreted and require pathologic confirmation of malignancy. Immune-mediated (non-malignant) lymphadenopathy which pathologically represents sarcoidal reaction can occur after ICI (Table 2) (1). While rare, ICI can cause immune cells to infiltrate lymph nodes or the tumor milieu, causing increases in tumor or node size radiographically (15). Hence, PET/CT alone may over-stage nodal false positivity after neoadjuvant ICI emphasizing the need for treatment response biomarkers.

Although PD-L1 expression and TMB have been associated with ICI efficacy in metastatic NSCLC trials, in phase II ICI-CT studies, these biomarkers and their correlation to predict ICI efficacy and tumor pathologic regression is variable (1). ctDNA has the ability to discover micrometastatic disease with potentially higher sensitivity than radiographic detection (16). In multiple studies, ctDNA levels could detect molecular residual disease (MRD) prior to radiographic recurrence, and ctDNA levels correlated with adjuvant treatment response $(16,17)$. ctDNA 
Table 2 Proposed grading system for intraoperative nonmalignant lymphadenopathy, peripheral (pleural) fibrosis, and perihilar/lobar or mediastinal adhesions*

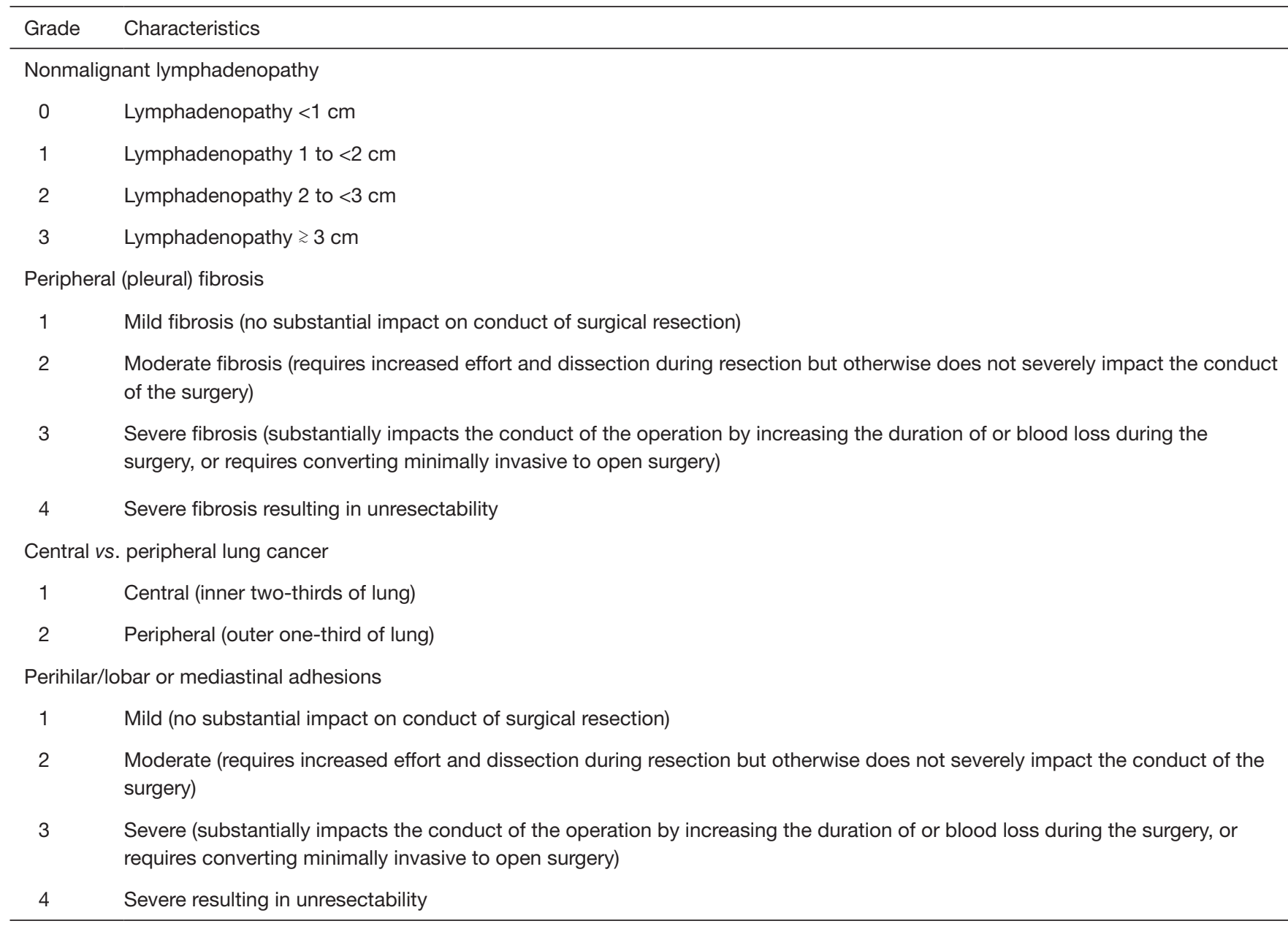

*, adapted from Lee et al. (2).

levels may also correlate with pathological responses. In the phase II study of neoadjuvant nivolumab, there was correlation between ctDNA clearance and VT reduction $(>30 \%)(18)$. These studies suggest that ctDNA could be a biomarker of neoadjuvant therapy response. However, ctDNA detection remains challenging in patients with early stage disease and optimization of assays is needed prior to using ctDNA outside of studies (1).

\section{Consensus 5: surgery can be performed 4-6 weeks after the last cycle of neoadjuvant immunotherapy}

Most phase II neoadjuvant trials report a window for surgery of 3-6 weeks after the end of 2-4 cycles of therapy (time endpoint D; Figure 1). However, duration from end of neoadjuvant therapy to surgery usually includes a protocol defined "washout period" to recover from drug toxicities (time endpoint C; Figure 1). This washout period is rarely reported which can mask delays to surgery and make interpretation of delays difficult.

Several studies have reported protocol defined delays to surgery (time endpoints A-C; Figure 1). In LCMC3, the washout period was 8 days, followed by window for surgery of 20 days (3). Only $12 \%$ of patients had surgery outside of protocol window which was mainly due to non-drug related issues. In CM816, median time from last neoadjuvant dose to surgery was 5.3 weeks (5). The washout period was approximately 4 weeks. Delays to surgery were in $21 \%$ of patients, but only $4 \%$ had delays due to TRAEs (5). 
In ICI alone trials, surgical resection can theoretically occur immediately after last dose of neoadjuvant ICI given its attendant low toxicity profile and no need for a lengthy washout period. Trials of neoadjuvant CT-ICI have longer intervals (about 4-6 weeks) between completion of neoadjuvant therapy to surgery due to expectant high TRAEs, largely due to CT. To determine the optimal timing, transparent reporting of timelines from clinical trials is needed (2). The duration from end of neoadjuvant therapy to surgery, duration of washout period, and reasons for delay to surgery, must be reported to establish a recommended time-line from last dose of neoadjuvant therapy to resection (Figure 1).

\section{Consensus 6: there is no definitive evidence that neoadjuvant immunotherapy affects the conduct or safety of surgery}

The impact of neoadjuvant ICI on the conduct of surgery is controversial and poorly reported to date. There is no consensus whether neoadjuvant immunotherapy affects the conduct of surgery. In the NEOSTAR trial $40 \%$ of surgeries were deemed more complex than usual (14). They used a subjective 4-point scale, where a score of 2 was considered "normal dissection" and a score of 3 or more was "difficult dissection". However, most phase II trials have not prospectively collected data on the complexity of surgery. R0 resection may be a surrogate marker of neoadjuvant therapy efficacy, but also as an indirect measure of surgical complexity. R0 resection was 92\% (LCMC3) (3) and $83 \%$ (CM816) (5). There are clear limitations to crosstrial comparisons and the issue is further complicated by the differing percentage of stage III patients (63\% in CM816 vs. $49 \%$ in LCMC3) in trials which have expectant higher $\mathrm{R} 1 / \mathrm{R} 2$ resections.

Prospective data collection is needed to accurately understand the impact of neoadjuvant therapy on the conduct of surgery. We propose a grading system to assess intraoperative factors that measure complexity including non-malignant lymphadenopathy, peripheral (pleural) fibrosis, and peri-hilar/lobar or mediastinal adhesions (Table 2) (2). Additional important surgical endpoints include length of surgery (cut to close time), estimate blood loss, and conversion rates from minimally invasive to thoracotomy (Figure 1) (2).

We agree there is no definitive evidence that neoadjuvant immunotherapy affects the safety of surgery. Based on predominantly phase II neoadjuvant ICI trials, the incidence of grade $\geq 3$ TRAEs has been low (0-14\%) with ICI alone compared to combination ICI-CT (15-93\%) which was largely due to $\mathrm{CT}$ related neutropenia. In LCMC3, intra-operative vascular or bronchial injuries were rare. In Checkmate 816, TRAE grade $\geq 3$ was equivalent between both arms (34\% nivolumab and CT vs. 37\% CT alone) suggesting that ICI did not significantly add any serious TRAE to CT (5). Historically, neoadjuvant CT has a grade $\geq 3$ TRAE incidence of $12-60 \%(40-60 \%$ in most trials) (2). Additionally, pre-operative attrition (prevalence of patients not undergoing surgery) have been low in ICI trials $(0-18 \%)$ (1). Pre-operative attrition to surgery was $12 \%$ [LCMC3; $n=10 / 22$ due to progression of disease (PD)] (3), and $12-17 \%$ (CM816; nivolumab and CT arms) (5). Based on this data, there are no safety concerns with the use of preoperative ICI alone or CT-ICI.

\section{Consensus 7: pathological remission (MPR, pCR) needs to be assessed, recorded and reported by specialized pathologist after neoadjuvant immunotherapy}

The critical need to identify surrogate markers to OS in neoadjuvant or adjuvant clinical trials is heralded by the known prolonged time from patient enrollment to publication of 9-13 years (19). As a result, study results at time of publication may bear little relevance and the study drug may be obsolete. Following neoadjuvant CT, pathologic regression has been directly correlated with lung cancer mortality $(1,19,20)$. As a result, pathologic regression is a primary endpoint in many phase II or III trials of neoadjuvant ICI (1). However, the correlation between pathologic regression and survival has not been validated following neoadjuvant ICI. As such, the use of pathologic regression to assess preoperative ICI efficacy remains investigational until validation with a clinical endpoint (DFS, EFS, or OS). We agree that pathologic assessment of tumors should be conducted by a specialized pathologist after neoadjuvant immunotherapy. However differences in processing and assessment of specimens can lead to difficulties in interpretation and accuracy of MPR/ pCR measurement. The International Association for the Study of Lung Cancer (IASLC) recommends a standardized approach with assessment of VT, necrosis, and stroma (inflammation and fibrosis) to improve consistency of pathological assessment (21). Use of artificial intelligence 
(AI) to generate computed digital \%VT may standardize pathologic assessment. In LCMC3, digital and manual $\%$ VT were strongly correlated $(n=137, r=0.73)$ and digital \%VT demonstrated impressive predictability for manual MPR (AUROC $=0.975$ ) (22). Additionally, digital MPR was comparable to manual MPR in differentiating DFS rates (22). These promising results support AI feasibility use to standardize pathologic regression.

\section{Consensus 8: for neoadjuvant immunotherapy in nonprogressive patients, immunotherapy can be resumed after surgery and it can be maintained for 1 year}

There are six phase III neoadjuvant trials of neoadjuvant CT-ICI of which one study excludes adjuvant ICI (CM816; Table 1). To date, CM816 is the only phase III trial to report on MPR/pCR and safety $(4,5)$. But there has been no correlation to clinical outcome endpoints. However, there is precedent to offer adjuvant ICI alone outside of neoadjuvant strategies. IMpower010 demonstrated an impressive DFS advantage in resected stage II-IIIA NSCLC with $\mathrm{PD}-\mathrm{L} 1 \geq 1 \%$ with 1 year of atezoluzumab after adjuvant chemotherapy (10). The DFS benefit was most beneficial in PD-L1 TC $\geq 50 \%$, and TRAE grade $\geq 3$ was $21.8 \%(10)$. Whether patients who received neoadjuvant immunotherapy also need adjuvant immunotherapy is unclear. Perioperative ICI treatment is not without toxicities. In phase II trials, LCMC3 and NEOSTAR, one patient in each study died due to TRAE $(3,14)$. Validation of biomarkers are needed to better select patients that are most likely to benefit from immunotherapy. In the adjuvant setting, only patients with detectable ctDNA after chemoradiation had a response to consolidation ICI (17). Thus, ctDNA may help determine which patients could avoid potential adjuvant immunotherapy.

\section{Consensus 9: immunotherapy or induction chemotherapy can be offered in borderline resectable locally advanced NSCLC and consideration for surgery should be reevaluated upon restaging}

Treatment of stage III NSCLC should involve a multidisciplinary discussion. Standard of care for patients with unresectable stage III disease is concurrent platinumbased chemotherapy with radiotherapy followed by durvalumab in the absence of ICI contraindications.
The updated PACIFIC trial results showed that consolidation durvalumab after chemoradiation compared to chemoradiation alone was associated with improved 60-month OS, $42.9 \%$ and $33.4 \%$, respectively (23-25). While PACIFIC is practice changing, the study did not define the criteria of unresectable or inoperable stage III disease. As such, there is continued controversy of defining resectable $v s$. unresectable stage III NSCLC particularly given that the neoadjuvant CT-ICI trials allowed stage III enrollment. We recommend surgeon involvement in the treatment planning for stage III patients to determine resectability of tumors and operability of patients in multidisciplinary discussions.

\section{Acknowledgments}

Funding: None.

\section{Footnote}

Provenance and Peer Review: This article was commissioned by the editorial office, Translational Lung Cancer Research. The article did not undergo external peer review.

Conflicts of Interest: Both authors have completed the ICMJE uniform disclosure form (available at https:// dx.doi.org/10.21037/tlcr-21-830). JML reports grants and consulting fees from Astrazeneca, Bristol Myers Squibb, Genentech, Novartis; honoraria for lectures, presentations, speakers bureaus, manuscript writing or educational events from Astrazeneca, Genentech, Novartis; support from Astrazeneca, Genentech; Participation on a Data Safety Monitoring Board or Advisory Board from UCLA; stock from Moderna. CMF has no conflicts of interest to declare.

Etbical Statement: The authors are accountable for all aspects of the work in ensuring that questions related to the accuracy or integrity of any part of the work are appropriately investigated and resolved.

Open Access Statement: This is an Open Access article distributed in accordance with the Creative Commons Attribution-NonCommercial-NoDerivs 4.0 International License (CC BY-NC-ND 4.0), which permits the noncommercial replication and distribution of the article with the strict proviso that no changes or edits are made and the original work is properly cited (including links to both the formal publication through the relevant DOI and the license). 
See: https://creativecommons.org/licenses/by-nc-nd/4.0/.

\section{References}

1. Lee JM, Tsuboi M, Brunelli A. Surgical perspective on neoadjuvant immunotherapy in non-small cell lung cancer. Ann Thorac Surg 2021. [Epub ahead of print]. doi: 10.1016/j.athoracsur.2021.06.069.

2. Lee JM, Kim AW, Marjanski T, et al. Important surgical and clinical end points in neoadjuvant immunotherapy trials in resectable NSCLC. JTO Clin Res Rep 2021;2:100221.

3. Lee J, Chaft J, Nicholas A, et al. PS01.05 Surgical and Clinical Outcomes With Neoadjuvant Atezolizumab in Resectable Stage IB-IIIB NSCLC: LCMC3 Trial Primary Analysis. J Thorac Oncol 2021;16:S59-61.

4. Forde PM, Spicer J, Lu S, et al. Abstract CT003: Nivolumab (NIVO) + platinum-doublet chemotherapy (chemo) vs chemo as neoadjuvant treatment (tx) for resectable (IB-IIIA) non-small cell lung cancer (NSCLC) in the phase 3 CheckMate 816 trial. Cancer Research 2021;81:CT003.

5. Spicer J, Wang C, Tanaka F, et al. Surgical outcomes from the phase 3 CheckMate 816 trial: Nivolumab (NIVO) + platinum-doublet chemotherapy (chemo) vs chemo alone as neoadjuvant treatment for patients with resectable non-small cell lung cancer (NSCLC). J Thorac Oncol 2021;39:8503.

6. Huang M, O'Shaughnessy J, Zhao J, et al. Association of Pathologic Complete Response with Long-Term Survival Outcomes in Triple-Negative Breast Cancer: A MetaAnalysis. Cancer Res 2020;80:5427-34.

7. Mok TSK, Wu YL, Kudaba I, et al. Pembrolizumab versus chemotherapy for previously untreated, PD-L1expressing, locally advanced or metastatic non-small-cell lung cancer (KEYNOTE-042): a randomised, open-label, controlled, phase 3 trial. Lancet 2019;393:1819-30.

8. Kim JY, Kronbichler A, Eisenhut M, et al. Tumor Mutational Burden and Efficacy of Immune Checkpoint Inhibitors: A Systematic Review and Meta-Analysis. Cancers (Basel) 2019;11:1798.

9. Kwiatkowski DJ, Rusch VW, Chaft JE, et al. Neoadjuvant atezolizumab in resectable non-small cell lung cancer (NSCLC): Interim analysis and biomarker data from a multicenter study (LCMC3). J Thorac Oncol 2019;37:8503.

10. Wakelee HA, Altorki NK, Zhou C, et al. IMpower010: Primary results of a phase III global study of atezolizumab versus best supportive care after adjuvant chemotherapy in resected stage IB-IIIA non-small cell lung cancer (NSCLC). J Thorac Oncol 2021;39:8500.

11. Calles A, Riess JW, Brahmer JR. Checkpoint Blockade in Lung Cancer With Driver Mutation: Choose the Road Wisely. Am Soc Clin Oncol Educ Book 2020;40:372-84.

12. Oxnard GR, Yang JC, Yu H, et al. TATTON: a multi-arm, phase Ib trial of osimertinib combined with selumetinib, savolitinib, or durvalumab in EGFR-mutant lung cancer. Ann Oncol 2020;31:507-16.

13. Wu YL, Tsuboi M, He J, et al. Osimertinib in Resected EGFR-Mutated Non-Small-Cell Lung Cancer. N Engl J Med 2020;383:1711-23.

14. Cascone T, William WN Jr, Weissferdt A, et al. Neoadjuvant nivolumab or nivolumab plus ipilimumab in operable non-small cell lung cancer: the phase 2 randomized NEOSTAR trial. Nat Med 2021;27:504-14.

15. Fujimoto D, Yoshioka H, Kataoka $Y$, et al. Pseudoprogression in Previously Treated Patients with Non-Small Cell Lung Cancer Who Received Nivolumab Monotherapy. J Thorac Oncol 2019;14:468-74.

16. Chaudhuri AA, Chabon JJ, Lovejoy AF, et al. Early Detection of Molecular Residual Disease in Localized Lung Cancer by Circulating Tumor DNA Profiling. Cancer Discov 2017;7:1394-403.

17. Moding EJ, Liu Y, Nabet BY, et al. Circulating Tumor DNA Dynamics Predict Benefit from Consolidation Immunotherapy in Locally Advanced Non-Small Cell Lung Cancer. Nat Cancer 2020;1:176-83.

18. Reuss JE, Anagnostou V, Cottrell TR, et al. Neoadjuvant nivolumab plus ipilimumab in resectable non-small cell lung cancer. J Immunother Cancer 2020;8:e01282.

19. Hellmann MD, Chaft JE, William WN Jr, et al. Pathological response after neoadjuvant chemotherapy in resectable non-small-cell lung cancers: proposal for the use of major pathological response as a surrogate endpoint. Lancet Oncol 2014;15:e42-50.

20. Pataer A, Kalhor N, Correa AM, et al. Histopathologic response criteria predict survival of patients with resected lung cancer after neoadjuvant chemotherapy. J Thorac Oncol 2012;7:825-32.

21. Travis WD, Dacic S, Wistuba I, et al. IASLC Multidisciplinary Recommendations for Pathologic Assessment of Lung Cancer Resection Specimens After Neoadjuvant Therapy. J Thorac Oncol 2020;15:709-40.

22. Dacic S, Travis WD, Giltnane JM, et al. Artificial intelligence (AI)-powered pathologic response (PathR) assessment of resection specimens after neoadjuvant 
atezolizumab in patients with non-small cell lung cancer: Results from the LCMC3 study. J Thorac Oncol 2021;39:106.

23. Spigel DR, Faivre-Finn C, Gray JE, et al. Fiveyear survival outcomes with durvalumab after chemoradiotherapy in unresectable stage III NSCLC: An update from the PACIFIC trial. J Thorac Oncol

Cite this article as: Faltermeier CM, Lee JM. Neoadjuvant immunotherapy in resectable non-small cell lung cancer at a checkpoint. Transl Lung Cancer Res 2021;10(12):4328-4335. doi: $10.21037 /$ tlcr-21-830
2021;39:8511.

24. Antonia SJ, Villegas A, Daniel D, et al. Overall Survival with Durvalumab after Chemoradiotherapy in Stage III NSCLC. N Engl J Med 2018;379:2342-50.

25. Antonia SJ, Villegas A, Daniel D, et al. Durvalumab after Chemoradiotherapy in Stage III Non-Small-Cell Lung Cancer. N Engl J Med 2017;377:1919-29. 